\title{
Clinical Aspects of Pulmonary Nontuberculous Mycobacteriosis
}

\author{
Hiroshi Moro and Toshiaki Kikuchi
}

\begin{abstract}
:
Nontuberculous mycobacterial (NTM) infections are an emerging problem. Common organisms include Mycobacterium avium, $M$. intracellulare, and $M$. kansasii, along with the $M$. avium intracellulare complex (MAC), which includes both M. avium and M. intracellulare. Typically, NTM infections affect the lungs and subsequently demonstrate a chronic course. Therefore, persistent respiratory symptoms generally indicate of the presence of pulmonary NTM diseases, and chest radiography, along with a sputum examination, are essential for its diagnosis. Because NTM are ubiquitous environmental organisms, a positive culture from a minimum of two separate expectorated sputum samples are required to make a diagnosis. The repertoire of effective drugs for treatment is considerably limited, indicating the need for long-term management with multiple drugs. Establishing a treatment regimen with high therapeutic efficacy and safety is an important issue for the future.
\end{abstract}

Key words: NTM, MAC, M. kansasii

(Intern Med Advance Publication)

(DOI: 10.2169/internalmedicine.4361-19)

\section{Overview}

Nontuberculous mycobacteria (NTM) include all mycobacteria except for Mycobacterium tuberculosis. Unlike tuberculosis, NTM etiologies are purely environmental, so patient isolation is not particularly important. Since these infections primarily affect the lungs, their clinical manifestations are characterized by cough, sputum, malaise, bloody sputum, and dyspnea. Patients may present with weight loss, fatigue, and appetite loss, indicating the presence of chronic inflammation. Common causative organisms include $M$. avium, $M$. intracellulare, $M$. kansasii, and the $M$. avium intracellulare complex (MAC), which includes both M. avium and M. intracellulare.

\section{The Diagnosis of NTM Lung Disease}

In 2007, the American Thoracic Society (ATS) and Infectious Disease Society of America (IDSA) jointly established a set of diagnostic criteria for NTM lung disease (1). The diagnosis is based on characteristic radiographic findings and positive culture results from at least two separate expectorated sputum samples, without the need for a clinical manifestation. The Japanese guideline, which was jointly established by the Japanese Society for Tuberculosis and Japanese Respiratory Society, recommends similar diagnostic criteria (2). In 2020, the ATS/European Respiratory Society (ERS)/European Society of Clinical Microbiology and Infectious Diseases (ESCMID)/IDSA guidelines were published, and the updated guidelines recommend the use of the same diagnostic criteria as before (3).

\section{Radiographic Findings}

Occasionally, an early diagnosis of asymptomatic NTM diseases may be established by chest radiography performed for screening purposes. However, the radiological findings of NTM lung diseases vary depending on the causative organism. Findings that are consistent with NTM lung disease on chest X-ray or high-resolution computed tomography (CT) include infiltration (usually nodular or reticular nodular), cavity, multifocal bronchiectasis, and/or multiple nodules (4). Although the presence of pleural exudate is particu- 


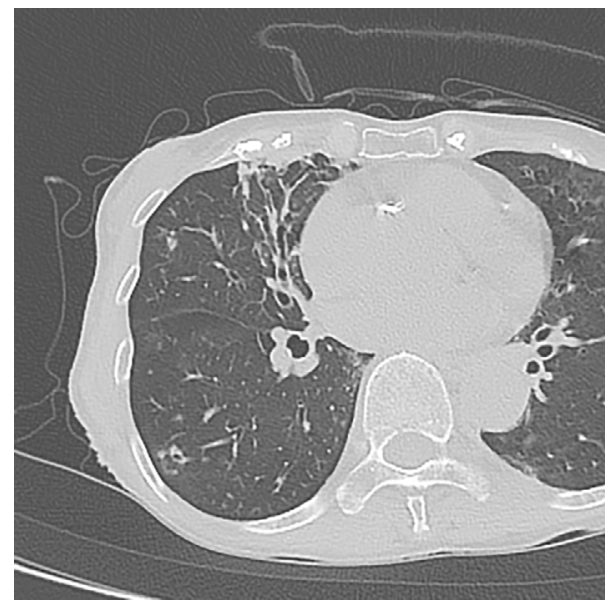

Figure 1. Nodular-bronchiectatic form of pulmonary MAC disease. High-resolution CT (1.0-mm collimation) of the right mid-lobe demonstrates dilated bronchi accompanied by scattered small nodules.

larly rare, reactive pleural thickening can often be seen.

Characteristic imaging findings suggest that pulmonary MAC diseases include the following two types: nodular/ bronchiectatic (NB; Figure) and fibrocavitary (FC; Figure 2) diseases, with the former considered to account for the majority of cases, being commonly observed among middleaged and older women without a history of smoking. A minimum of $50 \%$ of patients with MAC lung disease demonstrate radiographic abnormalities that are characterized by nodules associated with bronchiectasis or NB disease. Nodules and bronchiectasis are observed in the right middle lobe and left lingular segment (5). High-resolution CT of the chest is particularly useful for diagnosing this pattern of MAC lung diseases, since bronchiectasis and nodules are difficult to distinguish from simple chest radiographs (6). Despite the fact that the clinical course and prognosis of NB disease show considerable variation, the prognosis is relatively good. Radiological patterns of nodular/bronchiectatic properties are also observed in other NTM pathogens, such as M. abscessus.

FC disease is typically prevalent among elderly men with preexisting lung diseases, including emphysema and obsolete tuberculosis. FC disease generally progresses rapidly and is considered a risk factor with a poor prognosis, highlighting the importance of early intervention. Cavities generated by NTM pathogens tend to have thinner walls than those caused by tuberculosis $(7,8)$. M. kansasii- and $M$. szulgai-associated lung diseases exhibit clinical symptoms and imaging findings similar to those of pulmonary tuberculosis (e.g. cavities and nodules in the apex of the lung).

Pulmonary MAC diseases other than FC and NB types include the isolated nodule type, systemic dissemination type (9), and hypersensitive pneumonia type (10). Numerous factors, including host factors (11), pathogen virulence, and conditions of exposure, reportedly determine the type of disease.

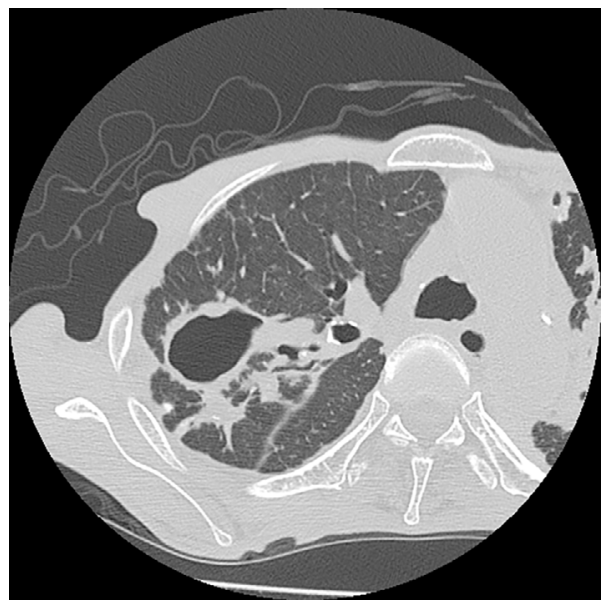

Figure 2. Fibrocavitary form of pulmonary MAC disease. High-resolution CT (1.0-mm collimation) shows a thin-walled cavity surrounded by homogeneous opacities in the right upper lobe.

\section{Performing a Microbiologic Evaluation}

Microbiological confirmation is essential for the diagnosis of NTM lung diseases. As NTM are ubiquitous environmental organisms, a positive sputum culture may be associated with environmental contamination or noninvasive colonization in patients with chronic lung disease. Therefore, positive culture results from at least two separate expectorated sputum samples are required for a diagnosis (1). Good-quality sputum is essential for both making a diagnosis and monitoring treatment efficacy. Sputum induction with hypertonic saline facilitates the acquisition of goodquality sputum in patients who are unable to expectorate sputum. Bronchial lavage obtained by bronchoscopy is not particularly affected by environmental contamination; therefore, the disease diagnosis can be confirmed through one or more positive culture results. Sample smear and cultivation should be simultaneously performed to ensure a prompt diagnosis; however, if smear results are positive and tuberculosis cannot be ruled out, it is important to consider an additional nucleic acid amplification method.

\section{Making a Serodiagnosis}

Studies have investigated the usefulness of serological diagnostic technology for diagnosing MAC diseases. A serologic diagnostic tool to detect IgA antibodies against MACspecific glycopeptidyl lipid core antigen using an enzyme immunoassay has been available in Japan since 2011. A study previously demonstrated favorable results for both the sensitivity and specificity in association with this assay (12). However, further research is needed to verify the utility of this technology. 


\section{Susceptibllity Testing}

While the usefulness of drug susceptibility tests for pulmonary NTM diseases has not been fully established, a drug susceptibility test method for NTM known as M24 has been established by the United States Clinical and Laboratory Standards Institute (13) and been adopted in the guidelines of both the US and Japan $(1,2)$. The international guidelines revised in 2020 (3) recommend treatment based on macrolide and amikacin susceptibility rather than empiric therapy for MAC lung disease. Similarly, for M. kansasii lung disease, treatment based on rifampicin susceptibility is recommended over empiric therapy.

\section{Treatment}

Treatment decisions are made by evaluating the expected risks and benefits of treatment. It is important to consider the immune status in immunocompromised hosts, and close observation should be performed. Treatment of pulmonary NTM infection depends on the infectious organism species. Generally, the repertoire of effective drugs is limited, suggesting the need for long-term management with multiple drugs.

\section{Pulmonary MAC Disease}

The timing of treatment is left to the discretion of the associated physician, and key components in the evaluation of patients are the clinical course, manifestation, imaging findings, and immune status, among other parameters. In brief, multidrug therapy including clarithromycin is essential for the treatment of this disease, based on the findings of previous studies (14-16). Although clarithromycin is considered a key drug, monotherapy should be strictly avoided due to concerns regarding drug-resistant strains (17).

The ATS/IDSA guideline (1) and ATS/ERS/ESCMID/ IDSA guideline (3) recommend a 3-drug, macrolide-based regimen for nodular bronchiectatic disease, including clarithromycin $(500 \mathrm{mg}$ twice per day), ethambutol $(25 \mathrm{mg} /$ $\mathrm{kg}$ per day), and rifampin $(600 \mathrm{mg}) 3$ times per week. A treatment regimen with daily doses of clarithromycin (500 $\mathrm{mg}$ twice per day), ethambutol (15 mg/kg per day), and rifampin $(10 \mathrm{mg} / \mathrm{kg}, 450 \mathrm{mg}$ to $600 \mathrm{mg}$ per day) is recommended for FC disease and severe nodular bronchiectatic disease. In addition, streptomycin or amikacin (10-15 mg/ $\mathrm{kg}, 3$ times per week) for the first 2 to 3 months should be considered in patients with FC disease, since these drugs are particularly effective for extracellular bacillus. In these regimens, clarithromycin and rifampin may be replaced by azithromycin and rifabutin, respectively. Dose modification is required for the elderly, and those with renal dysfunction and a low body weight to avoid adverse effects $(1,3,18)$, and the Japanese guideline recommends a similar treatment regimen with lower daily doses of clarithromycin (600 to
$800 \mathrm{mg}$ ) (19). However, the ATS/IDSA guideline and updated ATS/ERS/ESCMID/IDSA guideline recommends a minimum of one year of therapy after consecutive negative sputum cultures $(1,3)$, as there is limited evidence of the efficacy of these regimens and no consensus regarding the optimum duration for therapy. In addition, there is a lack of biomarkers for monitoring the therapeutic efficacy aside from sputum culture. Further investigations are thus required to determine the optimum therapeutic duration.

Because of the long-term nature of combination therapy for pulmonary MAC disease, side effects and drug interactions are of concern. To illustrate, rifampin reduces the blood concentration levels of clarithromycin $(20,21)$, which may lead to a decline in therapeutic efficacy. In addition, rifampin also modifies the levels of the corticosteroid administered to the patient. Considering this, a therapeutic regimen without rifampin is currently being explored. A previous study suggested that a two-drug regimen with clarithromycin and ethambutol can achieve clinical efficacy equivalent to or better than a three-drug regimen with rifampin (21). Although the international guidelines revised in 2020 continue to recommend triple therapy (3), treatment regimens that do not considerably burden patients are needed.

\section{M. kansasii Infection}

According to the US treatment guideline published in 2007, the standard regimen for treating M. kansasii disease includes rifampin $(600 \mathrm{mg} / \mathrm{day})$, isoniazid $(300 \mathrm{mg} / \mathrm{day})$, and ethambutol $(600 \mathrm{mg} / \mathrm{day})$ over a period of 12 months of negative sputum cultures (1). Generally, better therapeutic effects are expected with this disease than with pulmonary MAC diseases. However, pulmonary disease associated with M. kansasii may subsequently deteriorate in the absence of an appropriate treatment intervention, highlighting the importance of performing antimicrobial chemotherapy in all cases. One year after a negative sputum culture has been obtained is the recommended treatment duration, similar to that for pulmonary MAC diseases. Recurrence is typically associated with concern regarding the involvement of rifampin-resistant strains; however, considering their rarity during the initial treatment, there is no need to routinely perform a drug susceptibility test. The international guidelines revised in 2020 suggest that either isoniazid or macrolides should be used in combination with rifampicin and ethambutol (3).

\section{Surgical Management}

Conservative treatment primarily includes a clarithromycin-containing regimen; however, lung resection may be considered if drug therapy alone does not successfully eradicate the pathogens and associated symptoms. Several single-institution retrospective studies including a few patients suggested that a good treatment outcome may be attributed to surgical intervention $(22,23)$. The ATS/IDSA 
guideline suggests that surgical resection of limited (focal) disease in patients with a sufficient cardiopulmonary reserve to tolerate partial or complete lung resection can be successful when performed in combination with multidrug treatment regimens (1). There are no established criteria for patient selection; however, in cases refractory to drug therapy, with macrolide-resistant strains, or with serious complications, including hemoptysis, surgical intervention may be necessary. Considering the potentially severe perioperative complications, it is important to perform procedures in centers with expertise in both the medical and surgical management of mycobacterial diseases.

\section{The Prognosis}

A meta-analysis of HIV-negative patients with pulmonary MAC disease reported a treatment success rate of $38 \%$ in total patients (24). Furthermore, about $60 \%$ of patients with pulmonary MAC disease subsequently demonstrated consecutively negative sputum cultures with a standard regimen (25). However, a definitive treatment for pulmonary MAC disease has not yet been identified. Factors that predict a poor prognosis include FC form, positive sputum smear, and $M$. intracellulare as the causative organism (26).

In addition, studies have reported associations between the variable number of tandem repeats (VNTR) profiling data and the therapeutic response in patients with pulmonary MAC diseases (27). This method may thus be useful for predicting the therapeutic reactivity of pulmonary MAC diseases.

\section{Special Consideration Applicable to RA Patients}

Recent studies have determined that patients with rheumatoid arthritis (RA), and anti-TNF-alpha therapy are at an increased risk for NTM diseases. An epidemiological study performed in Asia revealed that the incidence of NTM diseases was 4.22 times greater in the RA group than in the non-RA group (28). The adjusted hazard ratio of NTM disease for the RA group was 4.17 (95\% confidence interval: 2.61-6.65). In addition, a study in North America revealed that the adjusted hazard ratio for NTM disease was 2.08 (95\% CI: 1.84-2.32) in the RA group, relative to the nonRA group (29). Furthermore, a review of the US Food and Drug Administration (FDA) Med Watch database for patients receiving anti-TNF-alpha therapy (30) showed that $44 \%$ of these patients had been diagnosed with NTM diseases. The median patient age was 62 years old; most patients $(65 \%)$ were women, and most $(70 \%)$ had RA. $M$. avium (50\%) was the most commonly implicated bacteria, and the pulmonary region was the most frequently reported site of disease $(56 \%)$.

The relatively frequent occurrence of pulmonary NTM disease in RA patients may be associated with preexisting structural abnormalities, such as those due to extraarticular involvement by RA. Therefore, pretreatment screening and appropriate management of NTM diseases are required in RA patients undergoing anti-TNF-alpha therapy. It is important to perform high-resolution CT before administering biological agents in order to evaluate the presence of airway disease. If the presence of NTM diseases is suspected, multiple sputum examinations or sampling by bronchoscopy should be considered. A serological assay is a promising technique in this context.

Furthermore, the imaging findings of airway diseases commonly complicating RA are similar to those of NTM disease on high-resolution CT, making it particularly difficult to differentiate between the two. In addition, colonization of NTM in the airway is not so very rare in RA patients; therefore, NTM diseases should be strictly diagnosed according to the diagnostic criteria in this setting. In principle, the use of biologics in patients with confirmed pulmonary NTM disease is contraindicated. However, in cases with NB forms of MAC lung disease, the use of biologics may be considered after carefully weighing the balance of the risks and benefits (31).

\section{The authors state that they have no Conflict of Interest (COI).}

\section{References}

1. Griffith DE, Aksamit T, Brown-Elliott BA, et al. An official ATS/ IDSA statement: diagnosis, treatment, and prevention of nontuberculous mycobacterial diseases. Am J Respir Crit Care Med 175: 367-416, 2007.

2. Nontuberculous Mycobacteriosis Control Committee of the Japanese Society for Tuberculosis. Guidelines for the diagnosis of pulmonary nontuberculous mycobacterial diseases--2008. Kekkaku: [Tuberculosis] 86: 37-39, 2011.

3. Daley CL, Iaccarino JM, Lange C, et al. Treatment of nontuberculous mycobacterial pulmonary disease: an official ATS/ERS/ESCMID/IDSA clinical practice guideline. Eur Respir J 56 (1): 2020.

4. Moore EH. Atypical mycobacterial infection in the lung: CT appearance. Radiology 187: 777-782, 1993.

5. Levin DL. Radiology of pulmonary Mycobacterium aviumintracellulare complex. Clin Chest Med 23: 603-612, 2002.

6. Koh WJ, Lee KS, Kwon OJ, Jeong YJ, Kwak SH, Kim TS. Bilateral bronchiectasis and bronchiolitis at thin-section CT: diagnostic implications in nontuberculous mycobacterial pulmonary infection. Radiology 235: 282-288, 2005.

7. Christensen EE, Dietz GW, Ahn $\mathrm{CH}$, et al. Initial roentgenographic manifestations of pulmonary Mycobacterium tuberculosis, M kansasii, and M intracellularis infections. Chest 80: 132-136, 1981.

8. Woodring JH, Vandiviere HM. Pulmonary disease caused by nontuberculous mycobacteria. J Thorac Imaging 5: 64-76, 1990.

9. Aoki A, Sakagami T, Yoshizawa K, et al. Clinical Significance of Interferon-gamma Neutralizing Autoantibodies Against Disseminated Nontuberculous Mycobacterial Disease. Clin Infect Dis 66: 1239-1245, 2018.

10. Daito H, Kikuchi T, Sakakibara T, et al. Mycobacterial hypersensitivity pneumonitis requires TLR9-MyD88 in lung CD11b+ CD11c + cells. Eur Respir J 38: 688-701, 2011.

11. Bamba $Y$, Moro $H$, Aoki N, et al. Multiplex cytokine analysis in Mycobacterium avium complex lung disease: relationship between CXCL10 and poor prognostic factors. BMC Infect Dis 19: 263, 
2019.

12. Kitada S, Kobayashi K, Ichiyama S, et al. Serodiagnosis of Mycobacterium avium-complex pulmonary disease using an enzyme immunoassay kit.. Am J Respir Crit Care Med 177: 793-797, 2008.

13. CLSI. Susceptibility testing of mycobacteria, Nocardia spp, and other aerobic actinomyces. 3rd ed. Clinical and Laboratory Standards Institute M24: 2018.

14. Wallace RJ Jr, Brown BA, Griffith DE, Girard WM, Murphy DT Clarithromycin regimens for pulmonary Mycobacterium avium complex. The first 50 patients. Am J Respir Crit Care Med 153 (6 Pt 1): 1766-1772, 1996.

15. Dautzenberg B, Piperno D, Diot P, Truffot-Pernot C, Chauvin JP. Clarithromycin in the treatment of Mycobacterium avium lung infections in patients without AIDS. Clarithromycin Study Group of France. Chest 107: 1035-1040, 1995.

16. Griffith DE, Brown BA, Cegielski P, Murphy DT, Wallace RJ Jr. Early results (at 6 months) with intermittent clarithromycinincluding regimens for lung disease due to Mycobacterium avium complex. Clin Infect Dis 30: 288-292, 2000.

17. Wallace RJ Jr, Brown BA, Griffith DE, et al. Initial clarithromycin monotherapy for Mycobacterium avium-intracellulare complex lung disease. Am J Respir Crit Care Med 149: 1335-1341, 1994.

18. Wallace RJ Jr, Brown BA, Griffith DE. Drug intolerance to highdose clarithromycin among elderly patients. Diagn Microbiol Infect Dis 16: 215-221, 1993.

19. Nontuberculous Mycobacteriosis Control Committee of the Japanese Society for Tuberculosis. Guidelines for chemotherapy of pulmonary nontuberculous mycobacterial disease--2012 revised version. Kekkaku: [Tuberculosis] 88: 29-32, 2013.

20. van Ingen J, Egelund EF, Levin A, et al. The pharmacokinetics and pharmacodynamics of pulmonary Mycobacterium avium complex disease treatment. Am J Respir Crit Care Med 186: 559-565, 2012.

21. Miwa S, Shirai M, Toyoshima M, et al. Efficacy of clarithromycin and ethambutol for Mycobacterium avium complex pulmonary disease. A preliminary study. Ann Am Thorac Soc 11: 23-29, 2014.

22. Nelson KG, Griffith DE, Brown BA, Wallace RJ Jr. Results of op- eration in Mycobacterium avium-intracellulare lung disease. Ann Thorac Surg 66: 325-330, 1998.

23. Shiraishi Y, Nakajima Y, Takasuna K, Hanaoka T, Katsuragi N, Konno H. . Eur J Cardiothorac Surg 21: 314-318, 2002.

24. Xu HB, Jiang RH, Li L. Treatment outcomes for Mycobacterium avium complex: a systematic review and meta-analysis. Eur J Clin Microbiol Infect Dis 33: 347-358, 2014.

25. van Ingen J, Ferro BE, Hoefsloot W, Boeree MJ, van Soolingen D. Drug treatment of pulmonary nontuberculous mycobacterial disease in HIV-negative patients: the evidence. Expert Rev Anti Infect Ther 11: 1065-1077, 2013.

26. Hayashi M, Takayanagi N, Kanauchi T, Miyahara Y, Yanagisawa T, Sugita Y. Prognostic factors of $634 \mathrm{HIV}$-negative patients with Mycobacterium avium complex lung disease. Am J Respir Crit Care Med 185: 575-583, 2012.

27. Kikuchi T, Kobashi Y, Hirano T, et al. Mycobacterium avium genotype is associated with the therapeutic response to lung infection. Clin Microbiol Infect 20: 256-262, 2014.

28. Yeh JJ, Wang YC, Sung FC, Kao CH. Rheumatoid arthritis increases the risk of nontuberculosis mycobacterial disease and active pulmonary tuberculosis. PloS one 9: e110922, 2014.

29. Brode SK, Jamieson FB, Ng R, et al. Risk of mycobacterial infections associated with rheumatoid arthritis in Ontario, Canada. Chest 146: 563-572, 2014.

30. Winthrop KL, Chang E, Yamashita S, Iademarco MF, LoBue PA. Nontuberculous mycobacteria infections and anti-tumor necrosis factor-alpha therapy. Emerg Infect Dis 15: 1556-1561, 2009.

31. Tokuda H, Harigai M, Kameda $\mathrm{H}$, et al. Consensus statements for medical practice: Biological agents and lung disease [Abridged English translation by the Japanese Respiratory Society]. Respir Investig 55: 229-251, 2017.

The Internal Medicine is an Open Access journal distributed under the Creative Commons Attribution-NonCommercial-NoDerivatives 4.0 International License. To view the details of this license, please visit (https://creativecommons.org/licenses/ by-nc-nd/4.0/).

(C) The Japanese Society of Internal Medicine Intern Med Advance Publication 